\title{
Time-dependent and independent effects of thyroid hormone administration following myocardial infarction in rats
}

\author{
IOANNA ILIOPOULOU ${ }^{1,2}$, IORDANIS MOUROUZIS ${ }^{1}$, GEORGE I. LAMBROU ${ }^{2,3}$, DIMITRA ILIOPOULOU ${ }^{2}$, \\ DIMITRIOS-DIONYSIOS KOUTSOURIS ${ }^{2}$ and CONSTANTINOS PANTOS ${ }^{1}$ \\ ${ }^{1}$ Department of Pharmacology, National and Kapodistrian University of Athens, Medical School, 11527 Athens; \\ ${ }^{2}$ Biomedical Engineering Laboratory, Department of Information Transmission Systems and Material Technology, \\ National Technical University of Athens, School of Electrical and Computer Engineering, 15780 Athens; \\ ${ }^{3}$ First Department of Pediatrics, University of Athens, Choremeio Research Laboratory, \\ National and Kapodistrian University of Athens, 11527 Athens, Greece
}

Received October 3, 2017; Accepted March 12, 2018

DOI: $10.3892 / \mathrm{mmr} .2018 .9008$

\begin{abstract}
Cardiac function is reduced following myocardial infarction (MI) due to myocardial injury and alterations in the viable non-ischemic myocardium, a process known as cardiac remodeling. The current treatments available for patients with acute MI (AMI) reduce infarct size, preserve left ventricular (LV) function and improve survival; however, these treatments do not prevent remodeling, which can lead to heart failure. The aim of the present study was to investigate the effects of thyroid hormone (TH) treatment following MI in an in vivo rat model. A total of 199 rats were separated into 3 groups: Sham operated and 2 different coronary artery ligation (CAL) groups. Rats subjected to CAL were randomly divided into a further 2 groups $24 \mathrm{~h}$ following surgery. The first group received standard rat chow (designated the CAL group), while the second group received food containing $0.05 \%$ thyroid powder (designated the CALTH group). The mean daily intake of $\mathrm{TH}$ per rat was estimated at $3.0 \mu \mathrm{g} \mathrm{T}_{3}$ and $12 \mu \mathrm{g} \mathrm{T}_{4}$. Echocardiography was used to monitor the rats. Large-scale analysis confirmed the favorable effects of $\mathrm{TH}$ treatment following CAL on various parameters of cardiac
\end{abstract}

Correspondence to: Professor Constantinos Pantos, Department of Pharmacology, National and Kapodistrian University of Athens, Medical School, Mikras Asias 75 Street, 11527 Athens, Greece

E-mail: cpantos@med.uoa.gr

Abbreviations: AMI, acute myocardial infarction; BW, body weight; CAL, coronary artery ligation; CALTH, coronary artery ligation with thyroid hormone treatment; DI, duration of intervention; ECG, electrocardiogram; LV, left ventricular; LVEF, LV-ejection fraction; LVESD, LV-end systolic diameter; LVLAD, LV-long-axis diameter; LVW, left ventricular weight; MHC, myosin heavy chain; SA, scar area; SI, sphericity index; SV, systolic velocity; SW, scar weight; TH, thyroid hormone; THT, thyroid hormone treatment; TR, thyroid hormone receptor; WTI, wall tension index

Key words: myocardial infarction, thyroid hormone, rat model, modeling function. TH treatment reduced LV dilation, and increased global and regional LV function. The development of cardiac hypertrophy was induced and, thus, wall stress was limited. Furthermore, TH treatment improved cardiac geometry, which manifested as an increased sphericity index. Myocardial function, as well as LV dilatation, following CAL and TH treatment was not closely associated with the extent of injury, indicating a novel therapeutic intervention that may alter the course of LV remodeling that typically leads to post-MI heart failure. Data modelling and regressions may be developed to enable the simulation of the pathophysiological processes that occur following MI, and to predict with accuracy the effects of novel or current treatments that act via the modulation of tissue injury, LV dilation, LV geometry and hypertrophy.

\section{Introduction}

Cardiac function is reduced following myocardial infarction (MI) due to myocardial injury and changes in the viable non-ischemic myocardium, a process known as cardiac remodeling. This response is characterized by the development of cardiac hypertrophy, altered cardiac chamber geometry, a shift in the expression of contractile proteins to a fetal pattern, a switch to glucose metabolism and the induction of fibrosis $(1,2)$.

Current treatments for patients with acute MI (AMI) reduce infarct size, preserve left ventricular (LV) function and improve survival. However, these therapies do not prevent remodeling, which may lead to heart failure $(3,4)$. In fact, $30-46 \%$ of patients fail to exhibit functional recovery until 6 months after AMI, despite the current treatments $(4,5)$. This requires further understanding of the pathophysiology of cardiac remodeling in order to comprehend the factors the contribute to disease progression, and to introduce novel effective treatments to repair the injured myocardium (6).

It has been well documented that thyroid hormone (TH) levels decrease following AMI $(3,7)$, in cardiac surgery (8) and in heart failure (9). The physiological significance of this response remains largely unknown and there is much controversy as to whether low T3 syndrome requires treatment (10). However, several observational studies showing 
an association between changes and clinical outcome have recently been presented, suggesting that it may be critical for the myocardial stress response. Indeed, experimental studies using cell and animal models have shown that $\mathrm{TH}$ receptor (TR) signaling is altered following myocardial ischemia or mechanical loading with important physiological consequences, while TH treatment may have beneficial effects (10). Our laboratory and others have shown that TH can favorably remodel the post-ischemic myocardium $(11,12)$. The effects of TH on cardiac remodeling after MI seem to be dose- and time-dependent, and were preserved in the presence of co-morbidities such as diabetes $(10,13-17)$. In rat models of MI, TH treatment promoted physiological remodeling of the non-ischemic myocardium, characterized by compensatory growth with favorable changes in the myosin heavy chain (MHC) expression pattern (decreased $\beta$-MHC and increased $\alpha$-MHC), ellipsoid reshaping and an improved LV ejection fraction $(\mathrm{EF} \%)(15,16)$.

Considering these previous findings, the purpose of the present study was to merge and analyze a large quantity of prior experimental data produced/published by our laboratory concerning the coronary artery ligation (CAL) model in rats at different time points, in order to provide a complete analysis that would allow us to delineate the process of myocardial remodeling and the effect of $\mathrm{TH}$ treatment. Towards that aim, we have assessed several myocardial parameters in rat experimental models using computational methodologies to achieve more in-depth study of the mechanisms of myocardial remodeling. The results of this analysis provided insights to better understand the favorable effect of TH treatment, which may assist efforts in translating this novel concept into clinical practice. Furthermore, the present study could be considered a first approach to creating a computational model to describe the phenomenon of myocardial remodeling under TH treatment.

\section{Materials and methods}

Animals. Animals were used in the present study as previously reported (15-17). The present study adhered to the principles of the Declaration of Helsinki regarding the ethical conduct of animal research, upheld by the World Medical Association. In addition, all experiments conformed to European legislation (European Union directive for the protection of animals used for scientific purposes 609/1986, revised in 2010/63/EU). The experimental protocols were approved by the Bioethics Committee of the National and Kapodistrian University of Athens Medical School (Prot. no. 1516013641/24/3/2010). The animals were administered humane care and housed in Plexiglas ${ }^{\circledR}$ chambers with ad libitum access to standard rodent pellet-diet and water at the Animal House of the Department of Pharmacology, University of Athens (license no. EL25 BIO 09). The laboratory conditions were maintained as optimal, in terms of temperature $\left(22-24^{\circ} \mathrm{C}\right)$, humidity $(35-65 \%)$ and light-to-dark cycles (12/12 h).

Experimental model and induction of MI. MI procedures were conducted as previously reported (15-17). In brief, rats were anesthetized with an intraperitoneal injection of ketamine (70 $\mathrm{mg} / \mathrm{kg})$ and midazolame $(0.1 \mathrm{mg} / \mathrm{kg})$, intubated and ventilated via a tracheal cannula using a constant-volume rodent ventilator (Inspira; 50 breaths/min; $1 \mathrm{ml} / 100$ gr tidal volume; Harvard Apparatus, Cambridge, MA, USA). Anesthesia was maintained by inhalation of small doses of sevoflurane (1-2\%). Left thoracotomy was performed at the fourth intercostal space, followed by pericardiotomy. The left coronary artery was then ligated with a 6-0 silk round-bodied suture. The heart was quickly returned to the chest cavity, the chest was closed and the rats were allowed to recover using assist mode ventilation. The recovery period was around 30-40 min. Atelectasis was prevented by using positive end-expiratory pressure at the end of the surgical procedure. A continuous electrocardiogram (ECG) was used to monitor heart beats and ECG ischemic changes following CAL. Body temperature was maintained at $37^{\circ} \mathrm{C}$ with a heating blanket (Harvard Homeothermic Blanket; 50-7061; Harvard Apparatus). The mortality rate was recorded as 15-20\% in the MI group during the first $24 \mathrm{~h}$ following surgery. The animals were left to recover for 2, 4 or 13 weeks after MI in order to assess short-term, mid-term and long-term recovery of function. The same procedure was followed for sham-operated animals, but the coronary artery was not ligated.

TH administration. Rats subjected to CAL were randomly divided in two groups $24 \mathrm{~h}$ after the operation. The first group received standard rat chow (designated the CAL group), while the second group received food containing $0.05 \%$ thyroid powder (T1251, with $0.42 \mu \mathrm{g} / \mathrm{mg} \mathrm{T}_{3}$ and $1.7 \mu \mathrm{g} / \mathrm{mg} \mathrm{T}_{4}$; designated the CALTH group; Sigma-Aldrich; Merck KGaA, Darmstadt, Germany), as previously described $(15,16)$. The mean daily intake of $\mathrm{TH}$ per rat was estimated at $3.0 \mu \mathrm{g} \mathrm{T}_{3}$ and $12 \mu \mathrm{g} \mathrm{T}_{4}$. Sham-operated rats receiving standard rat chow were designated the SO group.

Echocardiography. Rats were sedated with ketamine hydrochloride $(100 \mathrm{mg} / \mathrm{kg})$ and heart function was evaluated by echocardiography, as previously described $(15,16)$. Short and long-axis images were acquired using a GE Vivid 7 Pro digital ultrasound system and a GE I13L probe at $14.0 \mathrm{MHz}$ (GE Healthcare Life Sciences, Chicago, IL, USA). A large number of consecutive measurements were performed and subsequently analyzed by two independent operators.

LV internal diameter in the diastolic phase (LVIDd), LVID in the systolic phase (LVIDs), LV posterior wall thickness in the diastolic phase (LVPWd), and the EF\% were all measured. EF\% was calculated using the Simpson's rule. EF\% was used to determine the global contractile LV function.

Wall tension index (WTI) was defined as the ratio [(LVIDd/2) x PW thickness], as previously described $(16,18)$. WTI was measured in order to indirectly assess myocardial wall stress. In addition, the sphericity index (SI), defined as the ratio of the maximum long axis (in $\mathrm{mm}$ ) to the maximum short axis (in $\mathrm{mm}$ ) of the $\mathrm{LV}$, was determined in order to assess LV geometry. All measurements were averaged for $\geq 3$ consecutive cardiac cycles.

Experimental procedure. Rats $(n=199)$ were divided into three groups: Those that underwent a sham-operation (SO; $n=75$ ); those that were subjected to CAL without $\mathrm{TH}$ treatment (CAL; $n=88$ ); and those that were subjected to CAL with 


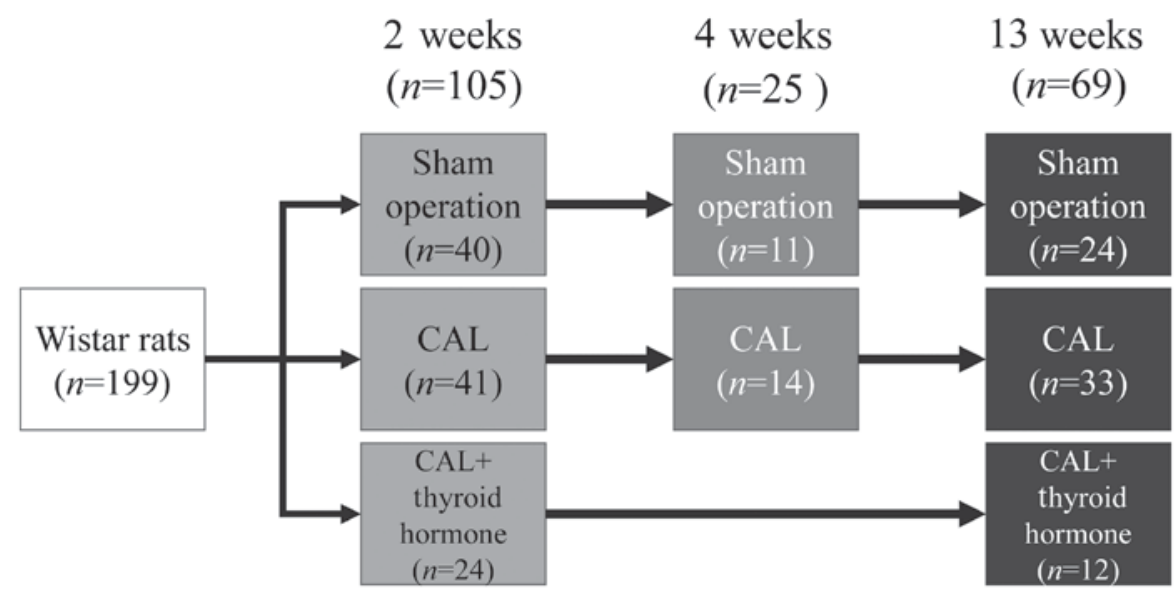

Figure 1. Diagrammatic representation of the experimental procedures followed during the present study. Rats were divided into 3 groups: Those that underwent an SO; those that were subjected to CAL without any TH treatment; and those that were subjected to CALTH. The SO and CAL experimental groups were monitored for 2, 4 and 13 weeks, respectively. The CALTH group was monitored for 2 and 13 weeks. SO, sham operation; CAL, coronary artery ligation; TH, thyroid hormone; CALTH, coronary artery ligation with thyroid hormone.

TH treatment (CALTH; $n=36)$. Rats in the SO and CAL experimental groups were monitored for $2(n=81), 4(n=25)$ or $13(n=69)$ weeks. Rats in the CALTH group were monitored for $2(n=24)$ or 13 weeks $(n=12)$. The experimental procedures are summarized in Fig. 1. At each time period following MI (2, 4 and 13 weeks), the rats were anaesthetized with ketamine hydrochloride and midazolam, subjected to echocardiography analysis and sacrificed while heart and lungs were removed.

Collected data and variables. Collected variables for each animal included the following: i) Surgery (intervention); ii) TH treatment; iii) duration of intervention; iv) LV-end diastolic diameter $(\mathrm{cm})$; v) LV-end systolic diameter $(\mathrm{cm})$; vi) LVPW thickness (cm); vii) long axis diameter $(\mathrm{cm})$; viii) LV EF\%; ix) systolic velocity (cm/sec); x) WTI(ratio); xi) SI (ratio); xii) heartbeats (beats per min); xiii) LV weight (mg); xiv) scar weight (SW) (mg); xv) scar area (SA) $\left(\mathrm{mm}^{2}\right)$. LV scar tissue was excised and the SA was measured in $\mathrm{mm}^{2}$ and the $\mathrm{SW}$ in mg. Variables are summarized in Table I.

Statistical analysis. Multiparameter analyses were performed with MATLAB ${ }^{\circledR}$ simulation environment (The MathWorks, Inc., Natick, MA, USA). One-, two- and three-way analysis of variance with a Bonferroni post hoc test were used to calculate the mean differences between groups. Continuous variables are expressed as the median \pm standard deviation, unless otherwise indicated. Correlations between variables were calculated using the Pearson's correlation coefficient. Linear regressions were performed using the $y=a x+b$ form and curves were estimated using a least-chi-squared approach. K-means, clustered scatter plots and hierarchical clustering algorithms were implemented using the MATLAB ${ }^{\circledR}$ simulation environment (The MathWorks, Inc.). The datasets used and/or analyzed during the current study are available from the corresponding author upon reasonable request.

\section{Results}

The time-independent effects of CAL (SO and CAL populations). CAL appeared to have a significant detrimental effect on several variables of cardiac function. In particular, LV EF\% (Fig. 2A), LV systolic velocity (cm/sec) (Fig. 2B), and SI (Fig. 2C) were significantly higher in the SO group when compared with those in the CAL group. In addition, indexes of cardiac remodeling such as LVEDD (cm) (Fig. 2D), LVESD (cm) (Fig. 2E), WTI (Fig. 2F) and LV weight (mg) (Fig. 2G) manifested as significantly higher in the CAL group when compared with those in the SO group. These results were predicted; the results also indicate the effects of CAL irrespective of time. Thus, it appears that there are time-independent effects of CAL.

The time-independent effects of TH treatment in the CALTH group. The time-independent effects of TH in the CAL group exhibited significant differences, being lower in the untreated group as compared with in the treated group with regard to LV EF\% (Fig. 3A), LVPW thickness (Fig. 3D), systolic velocity (Fig. 3E), SI (Fig. 3G) and heart beats (Fig. 3H). Conversely, significantly higher values were observed in the untreated group as compared with in the treated group with regard to the LVEDD (Fig. 3B), LVESD (Fig. 3C) and WTI (Fig. 3F).

The time-dependent effects in the CAL group. We studied the effect of treatment over time in the CAL group. LV EF\% significantly deteriorated between 2 and 4 weeks post-treatment, and was further reduced at 13 weeks (Fig. 4A). The LV diastolic diameter and systolic diameter significantly increased between 2 and 4 weeks, but not between 4 and 13 weeks at sacrifice (Fig. 4B and C). LVPW thickness significantly increased only after 13 weeks (Fig. 4D). WTI reached a maximum value at 4 weeks as compared with 2 and 13 weeks (Fig. 4E). SI reached its lowest value at 13 weeks, compared with at 2 and 4 weeks (Fig. 4F). A similar trend was observed for systolic velocity, with significant differences being observed between 2 and 4 weeks at sacrifice, and between 4 and 13 weeks at sacrifice (Fig. 4G). Finally, LV weight exhibited an increasing linear behavior with respect to time, with significant differences observed between 2 and 13 weeks at sacrifice, as well as at 4 and 13 weeks at sacrifice (Fig. 4I). 


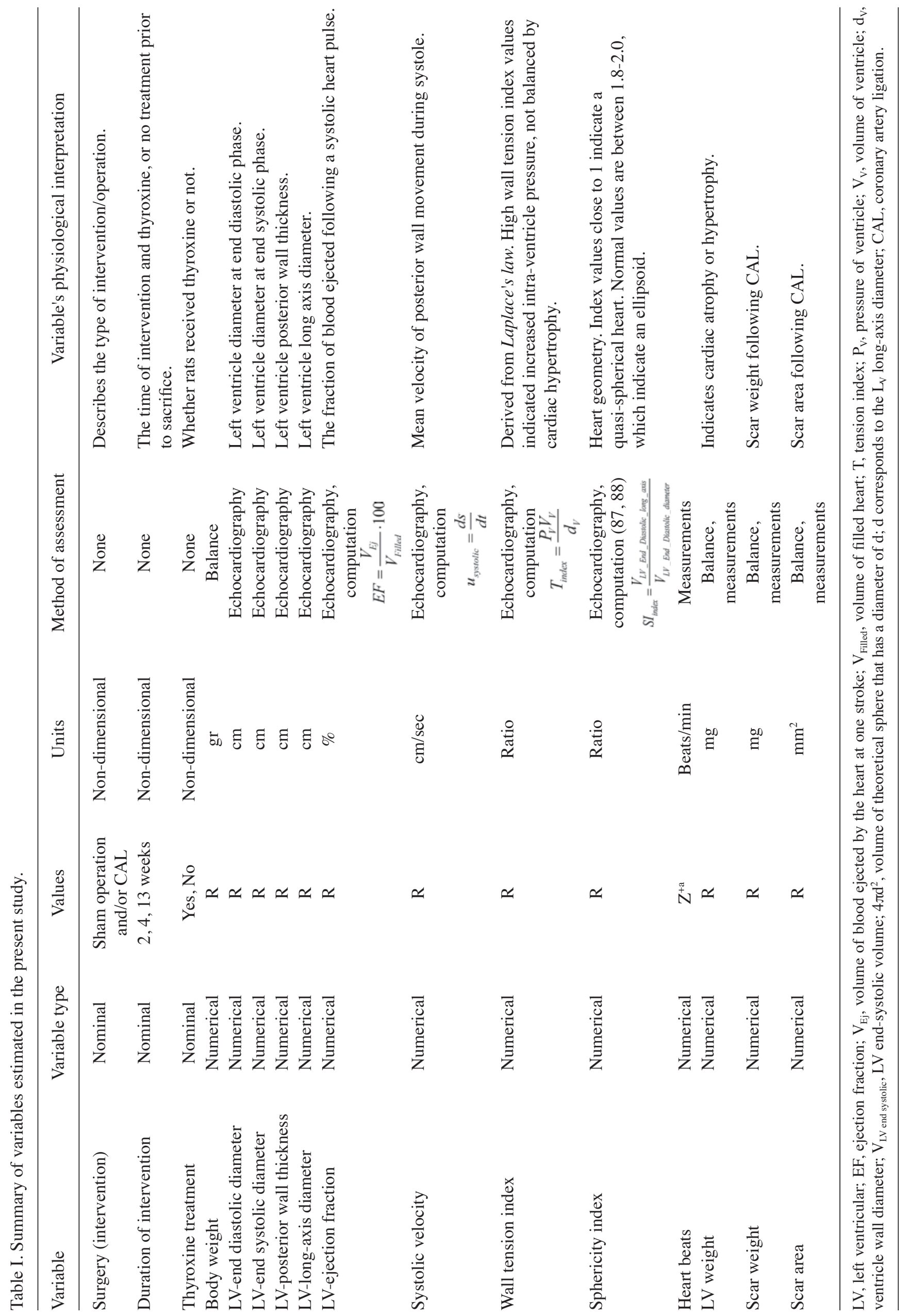



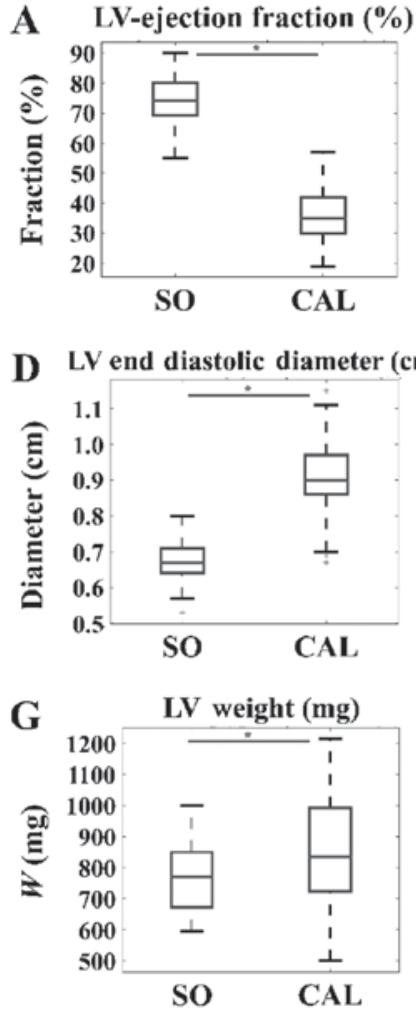
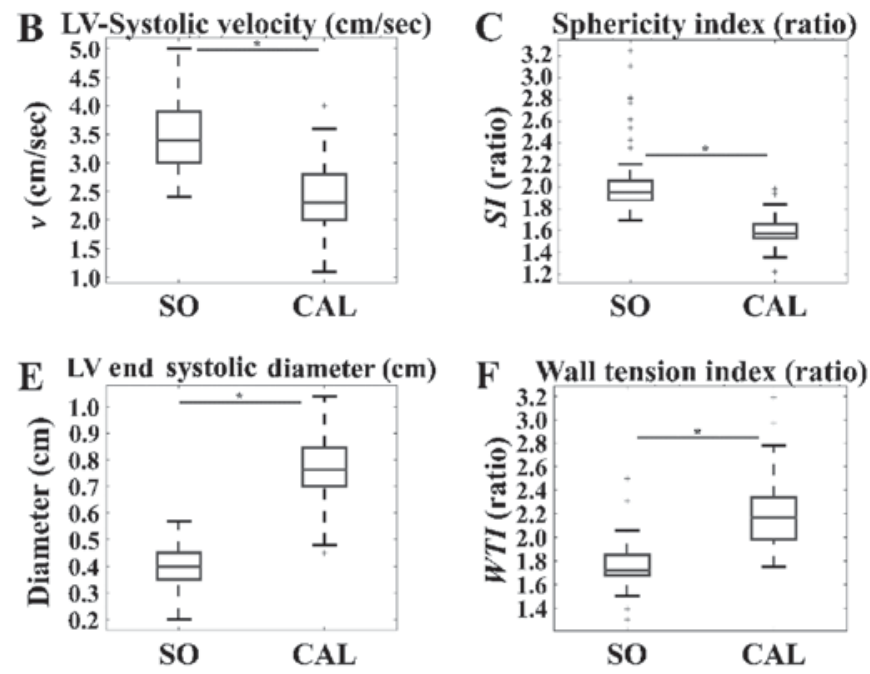

Figure 2. Effects of intervention in SO and CAL populations that did not receive thyroid hormone, irrespective of time. (A) LV ejection fraction (\%), (B) LV systolic velocity and (C) SI manifested to a significantly higher level in the SO group when compared with the CAL group. On the other hand, (D) LV end diastolic diameter, (E) LV end systolic diameter, (F) wall tension index and (G) LV weight were significantly higher in the CAL group when compared within the SO group. ${ }^{*} \mathrm{P}<0.05$, as indicated. SO, sham operation; CAL, coronary artery ligation; LV, left ventricular; SI, sphericity index; SO, sham operation; w, weight.

The time-dependent effects of TH treatment in the CAL group. LV EF\% appeared to be higher in CALTH rats as compared within CAL rats without TH treatment. Notably, no significant difference was observed in the EF\% between 2 and 13 weeks in CALTH rats (Fig. 4A). Similar behavior, as in the case of EF\%, was observed with systolic velocity (Fig. 4G). At 2 weeks there was no difference in the LV diastolic rats when compared with that in CAL rats without $\mathrm{TH}$, whereas the LV diastolic diameter at 13 weeks was significantly lower in CALTH rats (Fig. 4B). There was no significant difference in $\mathrm{LV}$ diastolic diameter between 2 and 13 weeks in the CALTH rats (Fig. 4B). LV systolic diameter was significantly higher in the CAL group without $\mathrm{TH}$ treatment when compared with that in the CALTH group at both 2 and 13 weeks. As with the diastolic diameter, there was no significant difference in the LV systolic diameter of CALTH rats between 2 and 13 weeks (Fig. 4C). LVPW thickness was significantly increased at 2 weeks in CALTH rats, as compared with in CAL rats without TH treatment, but no difference was observed at 13 weeks (Fig. 4D). Furthermore, WTI was significantly lower in the CALTH group compared within the CAL group without TH treatment at both 2 and 13 weeks (Fig. 4E). Heart beats was observed to be lower at 2, 4 and 13 weeks following CAL without TH treatment, as compared with the 2 and 13 weeks in CALTH rats (Fig. 4H). Finally, at 2 weeks, the LV weight exhibited a significant increase in the CALTH group compared within the CAL without $\mathrm{TH}$ treatment group, while no difference was observed at 13 weeks (Fig. 4I).
Notably, we found significant differences with respect to SW and SA within the CAL and CALTH groups. In particular, significant increases were observed in both SW and SA at 13 weeks, as compared with at 2 weeks, in the CAL group without TH treatment (Fig. 5). Furthermore, SA and SW were lower in CALTH rats compared within CAL rats without TH treatment at 13 weeks (Fig. 5).

Time-independent Pearson's correlation of variables in the group without TH treatment. Pearson's correlation analysis revealed parameters that manifested a rho value of $>0.8$ within the group that did not receive TH. In particular, it appeared that a positive correlation was present between LVSD and LVEDD ( $r h o=0.97)$, WTI and LVEDD ( $r h o=0.86)$, and SA, SW and LVEDD $(r h o=0.88$ and $r h o=0.82$, respectively), as well as between SA and SW with respect to LVSD ( $r h o=0.91$ and $r h o=0.85$, respectively). Conversely, it appeared that a negative correlation (rho<-0.8) was present between the LV EF\% and LVEDD (rho=-0.91), LV EF\% and LVSD (rho=-0.96), and SA and SW with respect to LV EF\% (rho $=0.92$ and $r h o=0.86$, respectively). Results are summarized in Table II.

Time-independent Pearson's correlation of variables in the group with TH treatment. Pearson's correlation analysis revealed parameters that manifested a rho value of $>0.8$ within the group that did receive TH. In particular, a positive correlation was present between LVSD and LVEDD (rho=0.96), WTI and LVEDD ( $r h o=0.87)$, and WTI and LVSD $(r h o=0.81)$. 

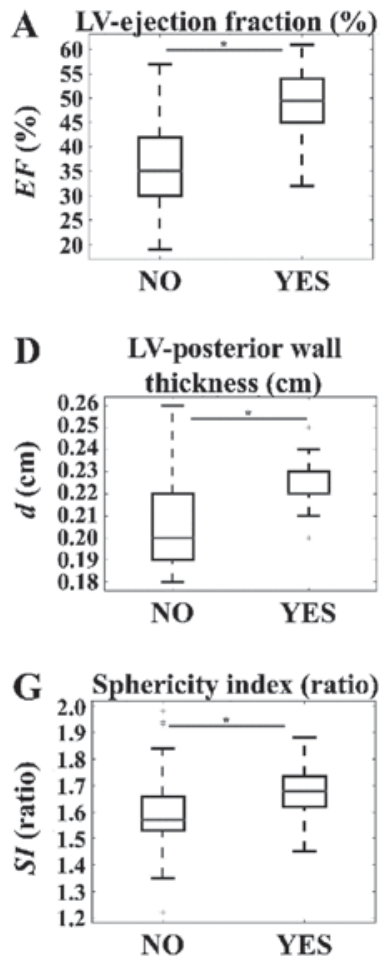
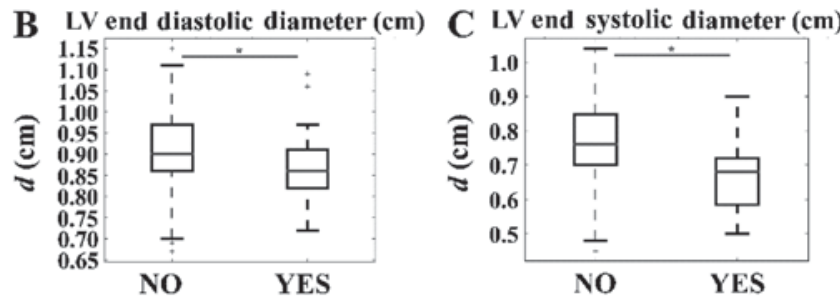

E Systolic velocity $(\mathrm{cm} / \mathrm{sec})$
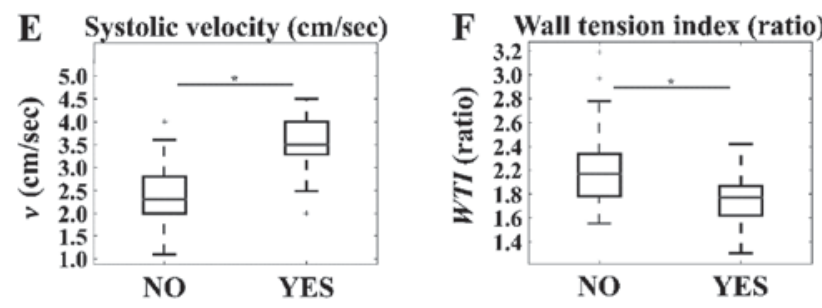

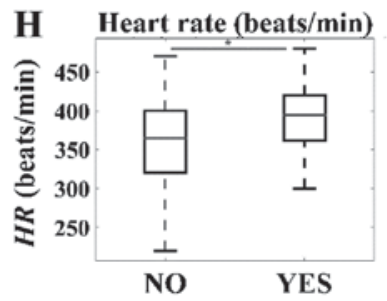

Figure 3. Time-independent effects of thyroid hormone in the CAL group. Significant reductions in the untreated group compared with the treated group were observed for (A) LV ejection fraction (\%), (D) LV posterior wall thickness, (E) systolic velocity, (G) sphericity index and (H) heart rate. On the other hand, significantly higher values were observed in the untreated group when compared with the treated group, for (B) LV end diastolic diameter, (C) LV end systolic diameter and $(\mathrm{F})$ wall tension index. " $\mathrm{P}<0.05$, as indicated. SO, sham operation; CAL, coronary artery ligation; LV, left ventricular; EF, ejection fraction; d, diameter; v, velocity; WTI, wall tension index; SI, sphericity index; HR, heart rate; NO, indicates CAL samples not treated with thyroid hormone; YES, indicates CAL samples that did receive thyroid hormone.

Conversely, a negative correlation (rho<-0.8) was present between LV EF\% and LVSD ( $r h o=-0.87$ ). Results are summarized in Table III.

Time-independent regressions of variables in all experimental groups. The time-independent regressions for $\mathrm{SA}, \mathrm{LV}$ EF\% and LVEDD with respect to the estimated variables in rats without $\mathrm{TH}$ intervention were estimated. The time-independent regression of SA, with respect to the estimated variables, indicated correlations of the ultrasound-estimated variables with the surgically estimated parameters. In particular, we found that SA, LV weight and LV EF\% manifested significant linear correlations with respect to the following variables: LV EF\% vs. SA $\left(R^{2}=0.84\right)$ (Fig. 6A); LVEDD vs. SA $\left(R^{2}=0.76\right)($ Fig. 6B); and LVESD vs. SA $\left(R^{2}=0.83\right)$ (Fig. $\left.5 C\right)$, irrespective of intervention (SO, CAL, CALTH) and the time of sacrifice. We also observed that the LV EF\% exhibited significant linear behavior with respect to the LVEDD $\left(R^{2}=0.82\right)$ (Fig. 6D). By contrast, the LV EF\% did not manifest significant linear correlation with respect to $\mathrm{LV}$ weight $\left(R^{2}=0.12\right)$ (Fig. $\left.6 \mathrm{E}\right)$, and neither did SI vs. SA $\left(R^{2}=0.46\right)$ (Fig. 6F) or systolic velocity vs. SA $\left(R^{2}=0.55\right)$ (Fig. $\left.6 \mathrm{G}\right)$.

Time-independent regressions of variables in the CAL experimental groups. The time-independent regressions for SA, LV EF\% and LVEDD with respect to other variables in rats with $\mathrm{TH}$ intervention were estimated. In contrast to the results obtained for rats without $\mathrm{TH}$ treatment, we found that the SA, LV weight and LV EF\% did not exhibit significant linear correlation with respect to the following variables: $\mathrm{LV}$ EF\% vs. SA $\left(R^{2}=0.48\right)$ (Fig. 7A); LVEDD vs. SA $\left(R^{2}=0.41\right)$ (Fig. 7B); and LVESD vs. SA $\left(R^{2}=0.40\right)$ (Fig. 7C), irrespective of intervention and the time of sacrifice. We also noted that LV EF\% did not manifest significant linear correlation with respect to the LVEDD $\left(R^{2}=0.58\right)$ (Fig. 7D) or LV weight $\left(R^{2}=0.02\right)$ (Fig. 7E), and neither did SI vs. SA $\left(R^{2}=0.33\right)$ (Fig. $7 \mathrm{~F})$ or systolic velocity vs. SA $\left(R^{2}=0.54\right)$ (Fig. $\left.7 \mathrm{G}\right)$. Finally, representative echocardiographic images from the different groups under investigation are presented in Fig. 8.

\section{Discussion}

The data included and analyzed in this study were produced in our laboratory over a period of 10 years, using rats subjected to CAL-induced AMI to study cardiac remodeling at different time-points. A number of rats were treated with $\mathrm{TH}$ in order to investigate its effects as a novel therapeutic approach. Changes in LV morphology and function were evaluated via echocardiography, and several measurements were included showing the deterioration of global LV function (EF\%), regional myocardial function (systolic velocity of PW), the development of LV dilation (LVEDD and LVESD) and hypertrophy (LV weight and PW thickness), as well as changes in LV geometry (SI) and WTI. We also included measurements that are considered to be important determinants of cardiac function, including the degree of cardiac injury (assessed based on SW and SA) and heart beats. 
A LV ejection fraction (\%)

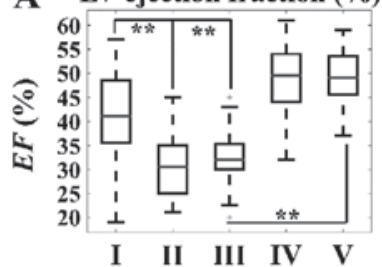

D LV posterior wall

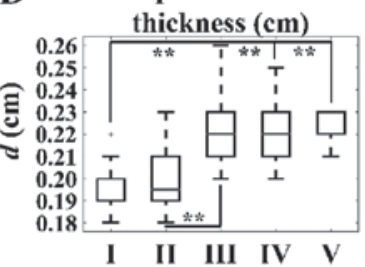

G Systolic velocity $(\mathrm{cm} / \mathrm{sec})$

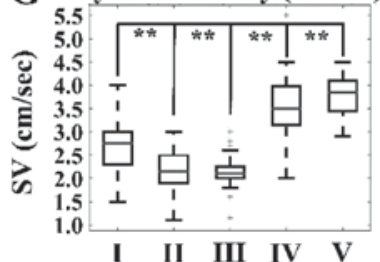

B LV end diastolic diameter $(\mathrm{cm})$

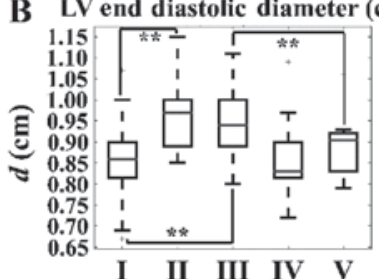

C LV end systolic diameter $(\mathrm{cm})$

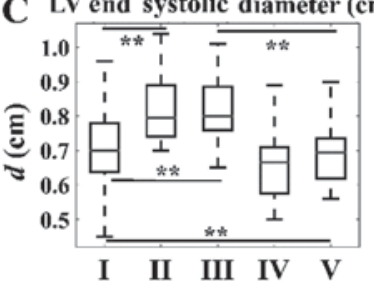

E Wall tension index (ratio)

F Sphericity index (ratio)
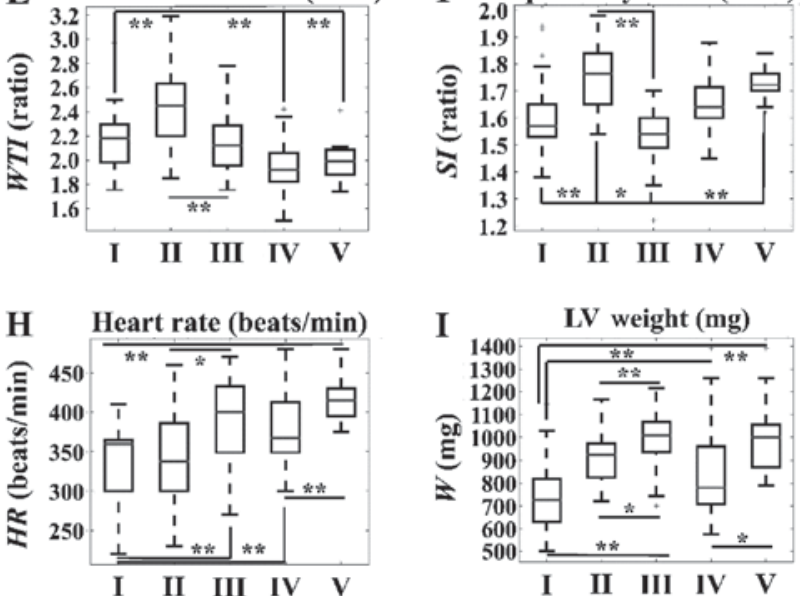

Figure 4. Time-dependent effects of TH in the CAL group. Significant differences were observed with respect to (A) LV ejection fraction, (B) LV end diastolic diameter, (C) LV end systolic diameter, (D) LV posterior wall thickness, (E) wall tension index, (F) sphericity index, (G) systolic velocity, (H) heart rate and (I) LV weight. " $\mathrm{P}<0.05$ and ${ }^{* *} \mathrm{P}<0.01$, as indicated. SV, systolic velocity; CAL, coronary artery ligation; LV, left ventricular; TH, thyroid hormone; d, diameter; WTI, wall tension index; w, weight; I, CAL group with no TH treatment at 2 weeks; II, CAL group with no TH treatment at 4 weeks; III, CAL group with no TH treatment at 13 weeks; IV, CAL group with TH treatment at 2 weeks; V, CAL group with TH treatment at 13 weeks.
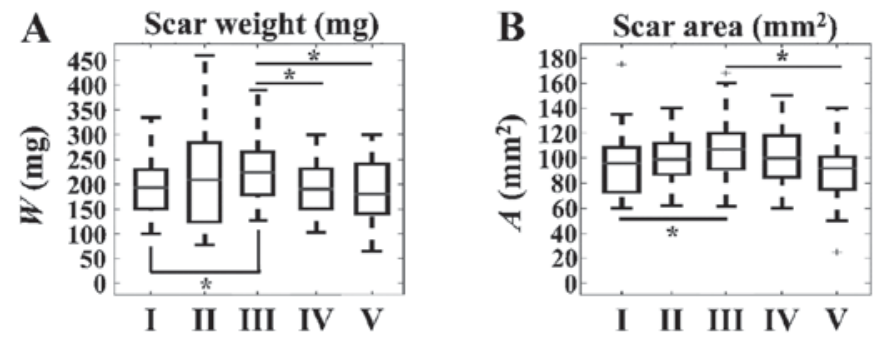

Figure 5. Time-dependent effects of TH in the CAL group with respect to scar parameters. Significant differences were observed with respect to (A) scar weight in the CAL group without TH treatment between 2 and 13 weeks. Significant differences were observed between the CAL group without TH treatment at 13 weeks and the CAL group with TH treatment at 2 weeks, as well as between the CAL group without TH treatment at 13 weeks and the CAL group with TH treatment at 13 weeks. (B) Significant differences were observed with respect to scar area in the CAL group without TH treatment between 2 and 13 weeks. In addition, significant differences were observed between the CAL group without TH treatment at 13 weeks and the CAL group with TH treatment at 13 weeks. ${ }^{*} \mathrm{P}<0.05$, as indicated. TH, thyroid hormone; CAL, coronary artery ligation; w, weight; A, area; I, CAL group with no TH treatment at 2 weeks; II, CAL group with no TH treatment at 4 weeks; III, CAL group with no TH treatment at 13 weeks; IV, CAL group with TH treatment at 2 weeks; V, CAL group with $\mathrm{TH}$ treatment at 13 weeks.

As expected, CAL resulted in LV dilation, which manifested as increased LVEDD and LVESD, and as reduced global and regional LV function. Furthermore, other manifestations of LV remodeling included the development of cardiac hypertrophy, increased wall stress and loss of the ellipsoid LV shape in favor of a spherical shape (manifested as a reduced SI). The time-dependent analysis of our results indicated a progressive nature of myocardial remodeling leading to end-stage heart failure. We found that global and regional LV function after MI progressively deteriorated over 2, 4 and 13 weeks, indicating that after the initial acute ischemic injury, the non-infarcted myocardium progressively deteriorates, leading to end-stage heart failure. In our experimental model, dilatation of the LV was shown to develop early, reaching a plateau at 4 weeks and then remaining stable between 4 and 13 weeks. Accordingly, pronounced LV dilatation is observed in patients with MI between 4 days and 4 weeks (19). Conversely, our data also showed that cardiac hypertrophy of the non-infarcted myocardium developed slowly over time and reached a maximum at 13 weeks post-MI. This mismatch between LV dilatation and LV hypertrophy may result in increased wall tension, particularly at 4 weeks after MI. Increased mechanical stress due to remodeling is hypothesized to exacerbate the series of maladaptive events leading to alterations of the contractile properties of the non-infarct zone (20). The development of cardiac hypertrophy at 13 weeks, despite its amelioration of wall tension, is a maladaptive response due to its delayed onset and association with unfavorable changes in MHC expression (more $\beta$-MHC and less $\alpha$-MHC) and calcium cycling proteins $(15,16)$, leading to a reduction in contractile function. Various approaches, including cardiac restraint devices and hydrogel injections, have been developed with the aim of potentially reducing mechanical stress post-MI (20). Furthermore, we reported that the LV geometry progressively deteriorated from an ellipsoid towards a spherical shape over time. Altered geometry per se can result in nearly a 50\% decrease in EF\% 


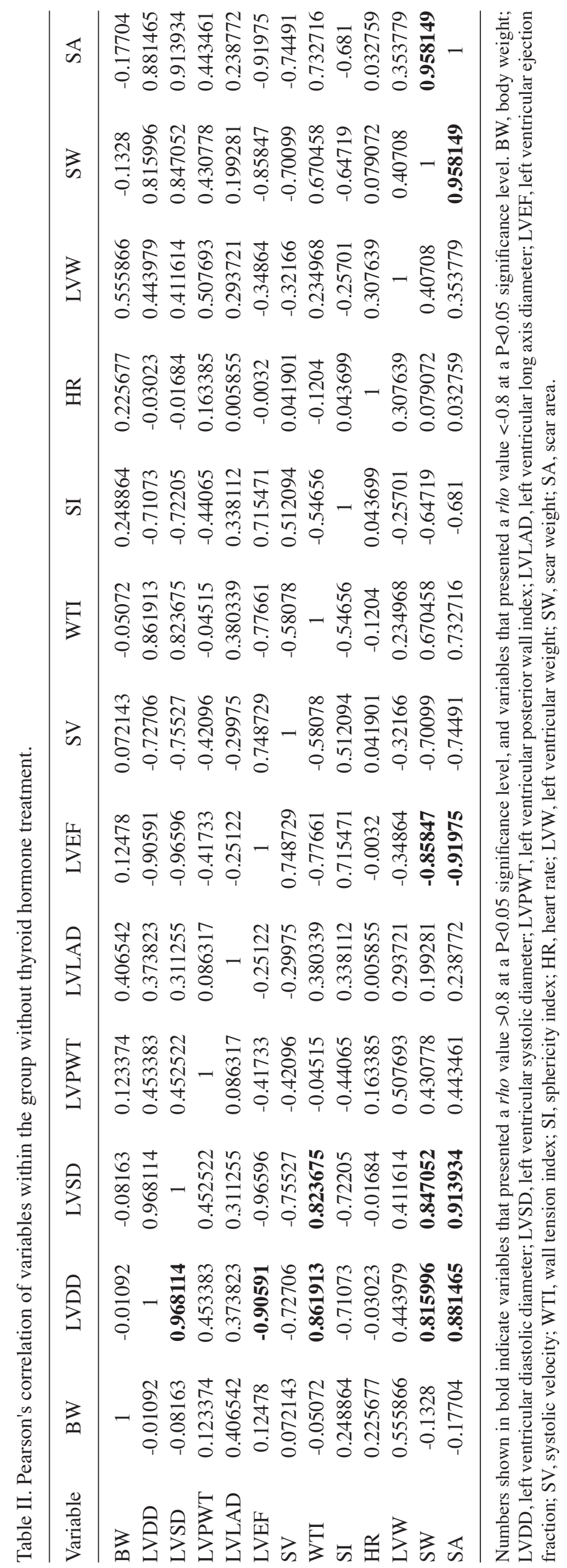




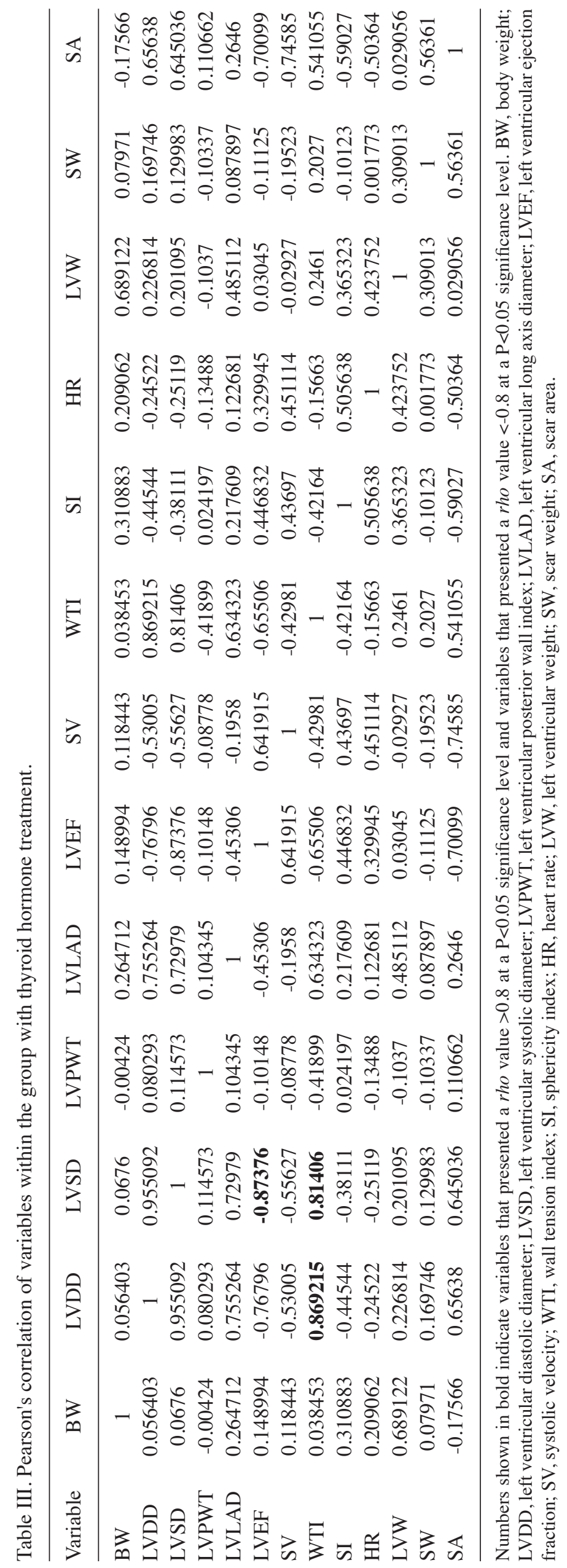




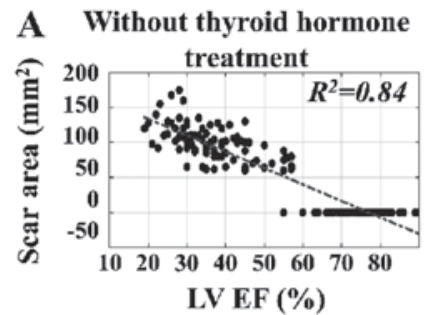

D Without thyroid hormone

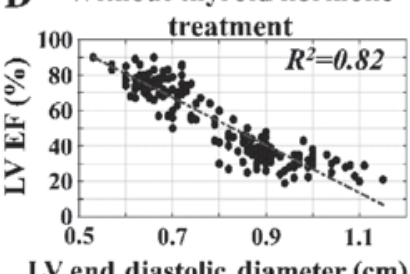

LV end diastolic diameter $(\mathrm{cm})$
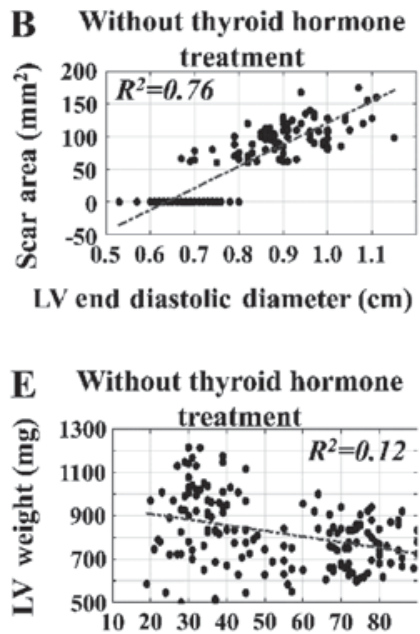

LV EF (\%)
C Without thyroid hormone

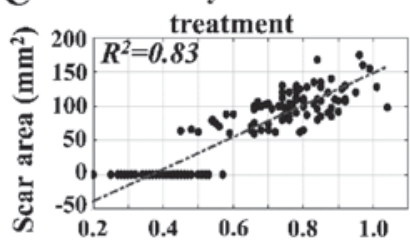

$\mathrm{LV}$ end systolic diameter $(\mathrm{cm})$

\section{F Without thyroid hormone}

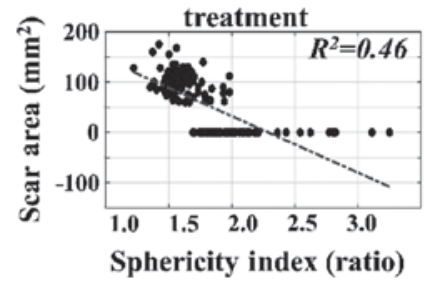

G Without thyroid hormone

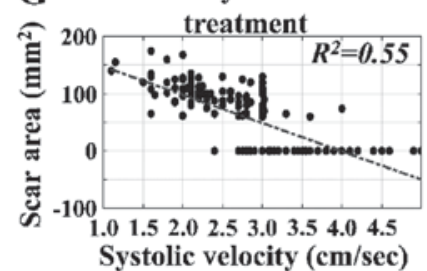

Figure 6. Time-independent regressions for SA with respect to estimated variables in rats without thyroid hormone intervention. The time-independent regression of SA with respect to the estimated variables indicated correlations between the ultrasound-estimated variables and the surgically estimated parameters. In particular, SA, LV weight and LV EF\% had markedly linear behavior with respect to the following variables: (A) $\mathrm{LV}$ EF\% vs. SA $\left(R^{2}=0.84\right)$, (B) LVEDD vs. SA $\left(R^{2}=0.76\right)$, and (C) LVESD vs. SA $\left(R^{2}=0.83\right)$, irrespective of the intervention and time of sacrifice. It was also observed that (D) LV EF\% manifested linear behavior with LVEDD $\left(R^{2}=0.82\right)$; (E) LV EF\% did not manifest significant linear behavior with respect to LV weight $\left(R^{2}=0.12\right)$; neither did (F) sphericity index vs. SA $\left(R^{2}=0.46\right)$ or $(\mathrm{G})$ systolic velocity vs. SA $\left(R^{2}=0.55\right)$. LV, left ventricular; EF, ejection fraction; SA, scar area; LVEDD, LV end diastolic diameter; LVESD, LV end systolic diameter.

and is associated with increased mortality (21), while it has been shown to predict LV remodeling after MI (22). Our results also indicate that the scar tissue expands over time (increased SA and weight at13 weeks compared with at 2 weeks), which is a characteristic of post-MI LV remodeling (19,20,22-24). Our large-scale analysis also revealed the favorable effects of $\mathrm{TH}$ treatment after CAL with respect to various parameters of cardiac function. We found that $\mathrm{TH}$ increases global and regional $\mathrm{LV}$ function after $\mathrm{MI}$, and no reduction in functional indices was observed between 2 and 13 weeks, showing that progressive deterioration of the non-infarcted myocardium following the initial acute ischemic injury is halted by $\mathrm{TH}$ administration. Furthermore, in our model, TH was found to allow the initial development of compensatory LV dilatation at 2 weeks, while inhibiting LV dilatation between 2 and 13 weeks after CAL. These results indicate that TH treatment inhibits the progressive development of LV remodeling that leads to heart failure. Similarly, TH treatment leads to the early development of cardiac hypertrophy at 2 weeks to match the early increase in LV dimensions, and normalizes wall tension as early as 2 weeks after MI, which indicates the compensatory nature of this hypertrophic response. In addition, no deterioration in mechanical stress, as measured by WTI, was observed between 2 and 13 weeks. The quality of this $\mathrm{TH}$-induced hypertrophic response has been shown to be different, since TH treatment promotes physiological growth of the non-infarcted myocardium, as characterized by compensatory hypertrophy with favorable changes in $\mathrm{MHC}$ expression (less $\beta$-MHC and increased $\alpha$-MHC) and calcium cycling proteins $(15,16)$. Similarly, in a mouse model of MI, we reported that $\mathrm{TH}$ replacement therapy restored functional recovery, and that this effect was associated with controlled activation of the pro-survival Akt signaling pathway, consistent with physiologic growth $(10,15,16)$. Furthermore, our analysis showed that TH treatment inhibits the development of unfavorable LV geometry changes that lead to a spherical ventricle. Thus, TH treatment helps to retain the ellipsoid shape of the LV over time, which is critical for contractile performance. This may be of therapeutic relevance, as the LV geometry appears to be an independent predictor of 10-year survival in patients with AMI (25).

It is noteworthy that the expansion of the scar tissue over time was not observed in TH-treated rats, which leads to a significant reduction in $\mathrm{SA}$ at 13 weeks between CAL and CALTH rats (25).

Regression analysis revealed that the observed deterioration of myocardial function in CAL rats (reduced $\mathrm{EF} \%$ ), as well as the extent of LV remodeling depicted by LV dilatation, was closely associated with the extent of the injury (SA). These findings are concordant with clinical observations showing that infarct extension assessed with magnetic resonance imaging has a strong negative association with myocardial systolic 
A With thyroid hormone

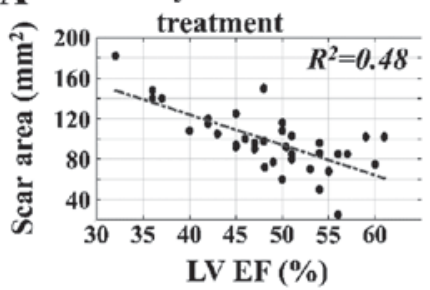

D With thyroid hormone

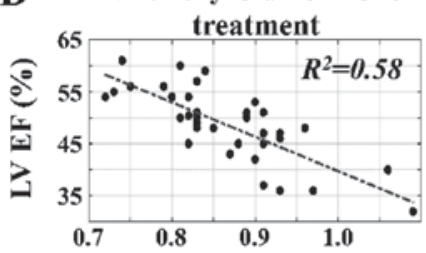

$\mathrm{LV}$ end diastolic diameter $(\mathrm{cm})$

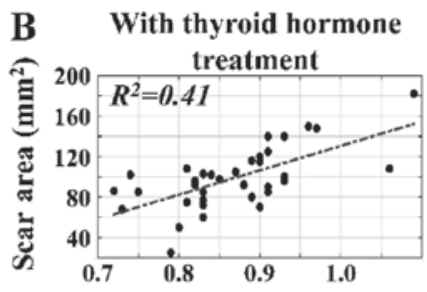

LV end diastolic diameter $(\mathrm{cm})$

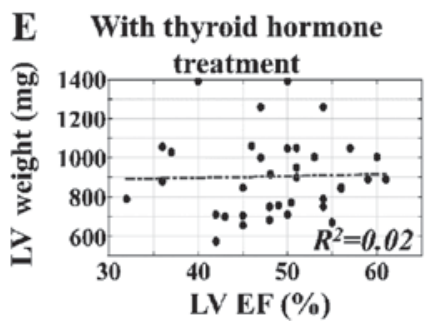

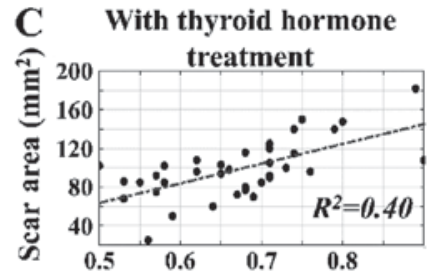

LV end systolic diameter (cm)

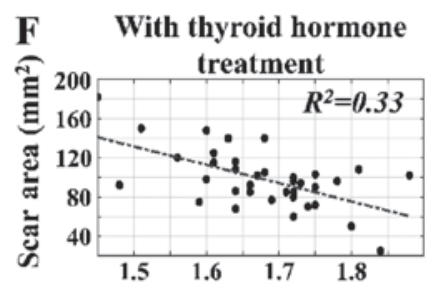

Sphericity index (ratio)

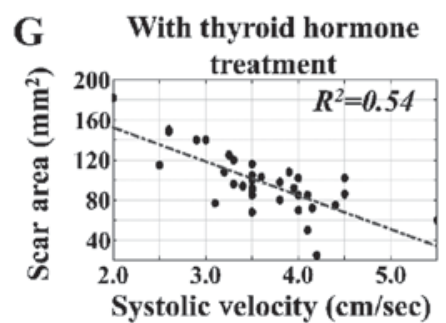

Figure 7. Time-independent regressions for SA with respect to estimated variables in rats with TH intervention. The time-independent regression of SA with respect to the estimated variables indicated correlations between the ultrasound-estimated variables and the surgically estimated parameters. In contrast to the results without TH treatment, SA, LV weight and LV EF\% did not exert significant linear behavior with respect to the following variables: (A) LV EF\% vs. SA $\left(R^{2}=0.48\right)$, (B) LVEDD vs. SA $\left(R^{2}=0.41\right)$, and (C) LVESD vs. SA $\left(R^{2}=0.40\right)$, irrespective of the intervention and time of sacrifice. It was also noted that LV EF\% did not have significant linear behavior with respect to (D) LVEDD $\left(R^{2}=0.58\right)$ or (E) LV weight $\left(R^{2}=0.02\right)(\mathrm{E})$, and $(\mathrm{F})$ neither did sphericity index vs. SA $\left(R^{2}=0.33\right)$ or $(\mathrm{G})$ systolic velocity vs. SA $\left(R^{2}=0.54\right)$. LV, left ventricular; TH, thyroid hormone; EF, ejection fraction; SA, scar area; LVEDD, LV end diastolic diameter; LVESD, LV end systolic diameter.

function (26). Furthermore, changes in the LV chamber dimensions during the first 6 months following MI in patients are dependent on the infarct size (27). To the best of our knowledge, our analysis showed for the first time that myocardial function, as well as LV dilatation, after CAL and TH treatment is not closely associated with the extent of injury, indicating that $\mathrm{TH}$ administration may represent a novel therapeutic intervention capable of modifying favorably the pathophysiology of post-MI development of heart failure $(26,27)$.

The present study could be considered as a first step in creating a computational model to describe the phenomenon of myocardial remodeling under TH treatment. Such modeling approaches could be developed to enable simulation of the pathophysiological processes following AMI, and to accurately predict the effects of novel and/or current treatments that act via modulation of tissue injury, LV dilation, LV geometry, hypertrophy and regional contractile function. Efforts have been made to simulate the post-infarction remodeling processes $(28,29)$. Successful implementation of such efforts, based on machine learning depends on a large number of inputs (systems that learn from data) (30). Data referred in the present study have been performed by our group using exactly the same experimental animal model for all reported experiments and the same equipment. A specific group of scientists has performed these experiments with a few additions over time. The problem of using different animals or reagents exists in all types of long-term experiments. This bias is consistent with the present measurements. Further on, comparisons reported in the present study included all measurements and in that sense they included the experimental bias from all previous experimental setups. Thus, significant differences observed appeared despite the timeand handling-related bias. Although the aforementioned factors could be considered as limitations of the study, our approach has included the systematic bias and in that sense conclusions could be drawn on the observed significant differences.

In conclusion, the novel findings from this large-scale analysis showed that post-AMI TH treatment: i) Improves LV function, but also inhibits the progressive deterioration over time of the non-infarcted myocardium and the progressive development of excessive LV dilatation; ii) inhibits expansion of the scar tissue over time and leads to smaller infarcts in the long-term; and iii) changes the pathophysiology of the development of heart failure, since parameters such as myocardial function and LV dilatation are not closely associated with the extent of the injury.

\section{Acknowledgements}

The authors would like to thank The National and Kapodistrian University of Athens, Medical School and The National Technical University of Athens, School of Electrical and Computer Engineering, Biomedical Engineering Laboratory (Athens, Greece) for their support during the present study. 

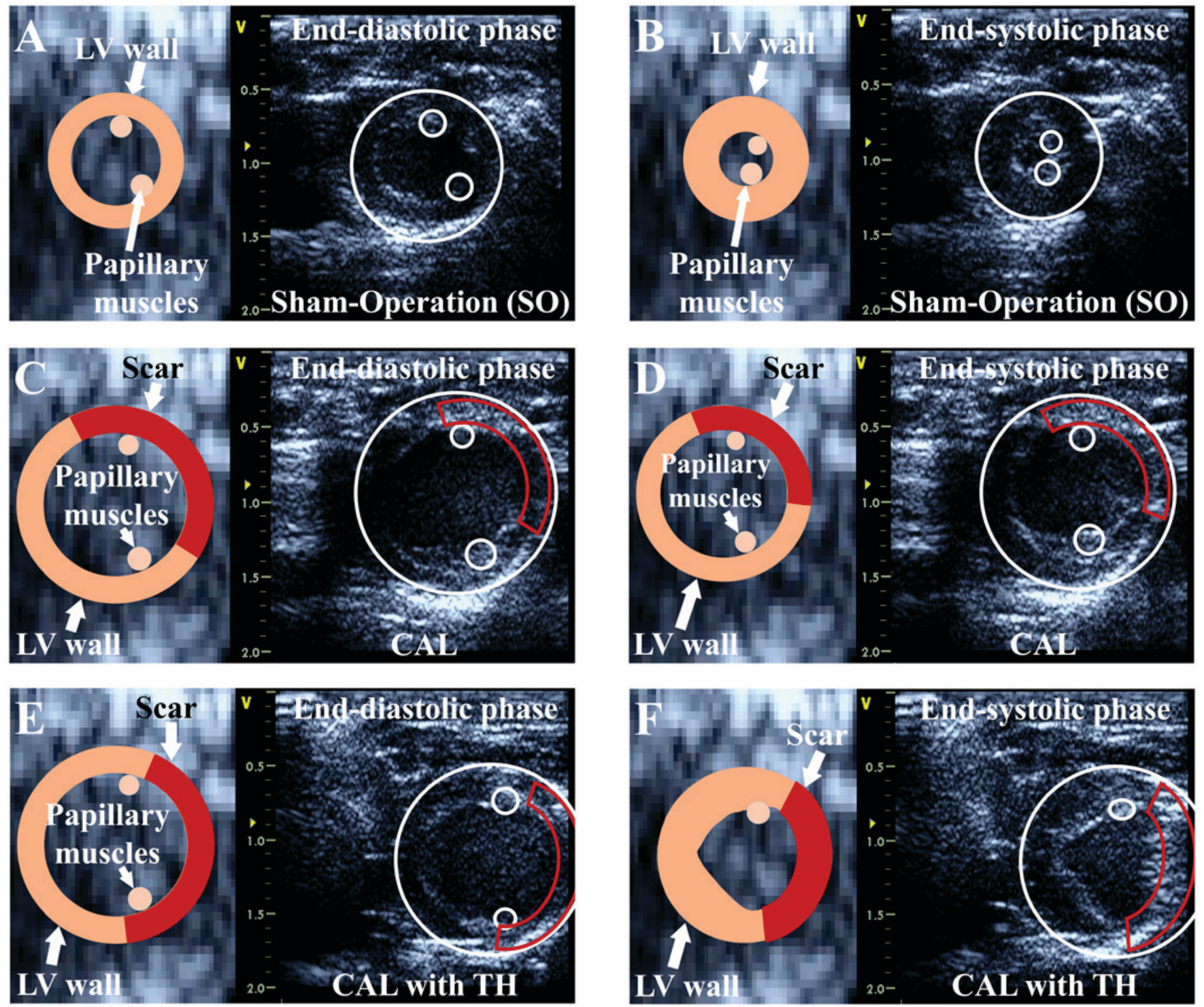

Figure 8. Representative echocardiographic images of parasternal short-axis view at the level of the papillary muscles during (A) the end-diastolic phase as well as the (B) end-systolic phase from rats subjected to SO. Echocardiographic images of the parasternal short-axis view at the level of the papillary muscles during the (C) end-diastolic phase as well as (D) the end-systolic phase from rats subjected to CAL without TH are presented. Finally, echocardiographic images of the parasternal short-axis view at the level of the papillary muscles during (E) the end-diastolic phase as well as (F) the end-systolic phase from rats subjected to CALTH. LV dilatation is evident in the groups with coronary artery ligation. The CALTH group is characterized by a greater development of LV hypertrophy and improved LV systolic function when compared with CAL without thyroid hormone. TH, thyroid hormone; LV, left ventricular; SO, sham operation; CAL, coronary artery ligation; CALTH, coronary artery ligation with thyroid hormone treatment.

\section{Funding}

No funding was received.

\section{Availability of data and materials}

The datasets used and/or analyzed during the current study are available from the corresponding author on reasonable request.

\section{Authors' contributions}

II collected the data and drafted the manuscript. IM collected the data, performed the experiments and data analysis, and drafted the manuscript. GIL performed data analysis and Neural Networks analysis, created the Matlab code and drafted the manuscript. DI contributed to the conception and design of the study, and drafted the manuscript. DDK was involved in the study conception and design, proof-editing the manuscript and gave final permission for publication. CP had substantial contribution to the conception and design of the work, provided the data and funding, proof-edited the manuscript and gave final permission for publication.

Ethics approval and consent to participate

The experimental protocols were approved by the Bioethics Committee of the National and Kapodistrian University of Athens Medical School (Athens, Greece; no. 1516013641/24/3/2010).

\section{Consent for publication}

Not applicable. 


\section{Competing interests}

The authors confirm that they have no competing interests.

\section{References}

1. Rajabi M, Kassiotis C, Razeghi P and Taegtmeyer H: Return to the fetal gene program protects the stressed heart: A strong hypothesis. Heart Fail Rev 12: 331-343, 2007.

2. Swynghedauw B: Molecular mechanisms of myocardial remodeling. Physiol Rev 79: 215-262, 1999.

3. Lymvaios I, Mourouzis I, Cokkinos DV, Dimopoulos MA Toumanidis ST and Pantos C: Thyroid hormone and recovery of cardiac function in patients with acute myocardial infarction: A strong association? Eur J Endocrinol 165: 107-114, 2011.

4. Springeling T, Kirschbaum SW, Rossi A, Baks T, Karamermer Y, Schulz C, Ouhlous M, Duncker DJ, Moelker A, Krestin GP, et al: Late cardiac remodeling after primary percutaneous coronary intervention-five-year cardiac magnetic resonance imaging follow-up. Circ J 77: 81-88, 2013.

5. Bolognese L, Neskovic AN, Parodi G, Cerisano G, Buonamici P, Santoro GM and Antoniucci D: Left ventricular remodeling after primary coronary angioplasty: Patterns of left ventricular dilation and long-term prognostic implications. Circulation 106: 2351-2357, 2002

6. Dargie H: Heart failure post-myocardial infarction: A review of the issues. Heart 91 (Suppl 2): ii3-6; discussion ii31, ii43-ii48, 2005.

7. Friberg L, Werner S, Eggertsen G and Ahnve S: Rapid down-regulation of thyroid hormones in acute myocardial infarction: Is it cardioprotective in patients with angina? Arch Int Med 162: 1388-1394, 2002.

8. Cerillo AG, Storti S, Clerico A and Iervasi G: Thyroid function and cardiac surgery: What should we measure and when? Ann Thorac Surg 89: 1010-1012, 2010.

9. Pingitore A, Landi P, Taddei MC, Ripoli A, L'Abbate A and Iervasi G: Triiodothyronine levels for risk stratification of patients with chronic heart failure. Am J Med 118: 132-136, 2005

10. Mourouzis I, Mantzouratou P, Galanopoulos G, Kostakou E, Roukounakis N, Kokkinos AD, Cokkinos DV and Pantos C: Dose-dependent effects of thyroid hormone on post-ischemic cardiac performance: Potential involvement of Akt and ERK signalings. Mol Cell Biochem 363: 235-243, 2012.

11. Pantos C, Mourouzis I and Cokkinos DV: New insights into the role of thyroid hormone in cardiac remodeling: Time to reconsider? Heart Fail Rev 16: 79-96, 2011.

12. Pantos C, Mourouzis I and Cokkinos DV: Rebuilding the post-infarcted myocardium by activating 'physiologic' hypertrophic signaling pathways: The thyroid hormone paradigm. Heart Fail Rev 15: 143-154, 2010.

13. Kalofoutis C, Mourouzis I, Galanopoulos G, Dimopoulos A, Perimenis P, Spanou D, Cokkinos DV, Singh J and Pantos C: Thyroid hormone can favorably remodel the diabetic myocardium after acute myocardial infarction. Mol Cell Biochem 345: 161-169, 2010.

14. Mourouzis I, Giagourta I, Galanopoulos G, Mantzouratou P, Kostakou E, Kokkinos AD, Tentolouris N and Pantos C: Thyroid hormone improves the mechanical performance of the post-infarcted diabetic myocardium: A response associated with up-regulation of Akt/mTOR and AMPK activation. Metabolism 62: 1387-1393, 2013.

15. Pantos C, Mourouzis I, Markakis K, Dimopoulos A, Xinaris C, Kokkinos AD, Panagiotou $\mathrm{M}$ and Cokkinos DV: Thyroid hormone attenuates cardiac remodeling and improves hemodynamics early after acute myocardial infarction in rats. Eur J Cardiothorac Surg 32: 333-339, 2007.
16. Pantos C, Mourouzis I, Markakis K, Tsagoulis N, Panagiotou M and Cokkinos DV: Long-term thyroid hormone administration reshapes left ventricular chamber and improves cardiac function after myocardial infarction in rats. Basic Res Cardiol 103 308-318, 2008.

17. Pantos C,Mourouzis I, Tsagoulis N, Markakis K, Galanopoulos G, Roukounakis N, Perimenis P, Liappas A and Cokkinos DV: Thyroid hormone at supra-physiological dose optimizes cardiac geometry and improves cardiac function in rats with old myocardial infarction. J Physiol Pharmacol 60: 49-56, 2009.

18. Grossman W, Jones D and McLaurin LP: Wall stress and patterns of hypertrophy in the human left ventricle. J Clin Invest 56: 56-64, 1975.

19. Gaudron P, Eilles C, Kugler I and Ertl G: Progressive left ventricular dysfunction and remodeling after myocardial infarction. Potential mechanisms and early predictors. Circulation 87: 755-763, 1993.

20. Holmes JW, Borg TK and Covell JW: Structure and mechanics of healing myocardial infarcts. Annu Rev Biomed Eng 7: 223-253, 2005.

21. Sallin EA: Fiber orientation and ejection fraction in the human left ventricle. Biophys J 9: 954-964, 1969.

22. Li F, Chen YG, Yao GH, Li L, Ge ZM, Zhang M and Zhang Y: Usefulness of left ventricular conic index measured by real-time three-dimensional echocardiography to predict left ventricular remodeling after acute myocardial infarction. Am J Cardiol 102: 1433-1437, 2008.

23. D'Elia N, D'Hooge J and Marwick TH: Association between myocardial mechanics and ischemic LV remodeling. JACC Cardiovasc Imaging 8: 1430-1443, 2015.

24. Kichula ET, Wang H, Dorsey SM, Szczesny SE, Elliott DM, Burdick JA and Wenk JF: Experimental and computational investigation of altered mechanical properties in myocardium after hydrogel injection. Ann Biomed Eng 42: 1546-1556, 2014.

25. Wong SP, French JK, Lydon AM, Manda SO, Gao W, Ashton NG and White HD: Relation of left ventricular sphericity to 10-year survival after acute myocardial infarction. Am J Cardiol 94: 1270-1275, 2004

26. Palazzuoli A, Beltrami M, Gennari L, Dastidar AG, Nuti R, McAlindon E, Angelini GD and Bucciarelli-Ducci C: The impact of infarct size on regional and global left ventricular systolic function: A cardiac magnetic resonance imaging study. Int J Cardiovasc Imaging 31: 1037-1044, 2015.

27. Konermann M, Sanner BM, Horstmann E, Grötz J, Laschewski F, Josephs W, Odenthal HJ and Sturm A: Changes of the left ventricle after myocardial infarction-estimation with cine magnetic resonance imaging during the first six months. Clin Cardiol 20: 201-212, 1997 .

28. Goktepe S, Abilez OJ, Parker KK and Kuhl E: A multiscale model for eccentric and concentric cardiac growth through sarcomerogenesis. J Theor Biol 265: 433-442, 2010.

29. Lee LC, Wall ST, Genet M, Hinson A and Guccione JM: Bioinjection treatment: Effects of post-injection residual stress on left ventricular wall stress. J Biomech 47: 3115-3119, 2014

30. Kourou K, Exarchos TP, Exarchos KP, Karamouzis MV and Fotiadis DI: Machine learning applications in cancer prognosis and prediction. Comput Struct Biotechnol J 13: 8-17, 2014.

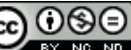

This work is licensed under a Creative Commons Attribution-NonCommercial-NoDerivatives 4.0 International (CC BY-NC-ND 4.0) License. 